\title{
Bcl2 inhibitor venetoclax +/- Anti-CD20: what do deep remissions mean?
}

\author{
Katharina T. Prochazka (D) - Barbara Uhl
}

Received: 29 July 2021 / Accepted: 16 November 2021 / Published online: 22 December 2021

(C) The Author(s) 2021

\begin{abstract}
Summary In recent years, treatment of patients exhibiting chronic lymphocytic leukemia has changed extensively due to advances in the development of targeted therapies. The Bcl-2 inhibitor venetoclax demonstrated outstanding results when used in mono- as well as combination therapy. Minimal residual disease (MRD) measurement has become an important endpoint in most studies and shows high prognostic potential. With upcoming combination strategies, the role of MRD measurement has also increased and is likely to become a routine marker in future clinical practice.
\end{abstract}

Keywords Chronic lymphocytic leukemia . Venetoclax · Bcl2 inhibitor · Remission · Minimal residual disease

Chronic lymphocytic leukemia (CLL) represents the most common form of leukemia in the western world [1]. The clinical course of patients is heterogeneous and is influenced by individual clinical factors and disease biology, including cytogenetic findings and IGHV mutational status, as well as alterations in the B cell receptor pathway and overexpression of antiapoptotic protein $\mathrm{Bcl} 2$, which was first described in 1993 by Hanada et al. with constitutive overexpression of the pro-survival protein Bcl2 [2-4]. The Bcl2inhibitor venetoclax has proven notable efficacy in the treatment of CLL, even in the relapsed/refractory

\section{K. T. Prochazka $(\varangle) \cdot$ B. Uhl}

Department of Haematology, Medical University Graz, Auenbruggerplatz 38, Graz, Austria

katharinatheresa.prochazka@uniklinikum.kages.at

B. Uhl

barbara.uhl@medunigraz.at setting including high-risk disease fludarabine-refractoriness, bulky adenopathy, TP53 abnormalities, and unmutated immunoglobulin heavy chain variable gene (IGHV), as well as in the first-line with or without the referred features [5-7]. Currently, there are two oppositional treatment strategies: prolongation of progression-free survival (PFS) without deep remissions using Bruton tyrosine kinase inhibitors in continuous therapy or time-limited treatment with venetoclax.

Since achievement of minimal residual disease (MRD) has been identified as prognostic factor for PFS and overall survival (OS) for patients receiving immunochemotherapy, its measurement in routine diagnosis and also as an endpoint in ongoing studies has increased substantially [8, 9]. In CLL, MRD is mostly determined by using at least 4-color flow cytometry (FLC), with the advantage of large availability and rapidness, at the expense of sensitivity at a level of $10^{-4}$. Availability of FLC in most centers allows widespread use, but standard operating procedures are required to allow multi-institutional studies. Polymerase chain reaction (PCR)-based methods include consensus, nested, and allele-specific oligonucleotide IGHV PCR methods. These techniques are more time and laboratory intensive but increase sensitivity up to $10^{-6}[10]$. Multiplex PCR, followed by sequencing to identify and quantify the signature immunoglobulin heavy and light-chain rearrangement of CLL cells can also be used as a next-generation sequencing approach (NGS). The advantages are the use of nonpatient-specific primers and the extremely high sensitivity (up to $10^{-6}$ ) [11]. Consequently, MRD analysis has been approved by the European Medicines Agency (EMA) as an intermediate/surrogate endpoint to assess treatment efficacy in randomized clinical trials designed to show superiority in terms of PFS in CLL patients [12]. Nevertheless, outside of clinical 
trials, MRD analysis has not yet been implemented in routine diagnostics by clinicians [13].

Various studies show that the compartment is critical, demonstrating higher rates of MRD in bone marrow than in peripheral blood $[14,15]$. Therefore, MRD negativity in peripheral blood always has to be confirmed by a bone marrow biopsy. However, for future clinical application of MRD assessment, it seems that the MRD evaluation will be sufficient by using peripheral blood. It could be shown that the MRD assessment in peripheral blood and bone marrow provided highly concordant results [15].

In a phase 1 study of venetoclax, 17 of 23 patients who achieved complete remission (CR) were evaluated for MRD negativity, using at least 4-color FLC [5]. Although the study population comprises patients with poor prognostic factors, 6 out of 17 patients tested negative for bone marrow MRD. In this study, patients with a partial remission (PR) have not been tested for MRD negativity, maybe underestimating the rate of negativity in this study.

Of 70 patients in a phase 2 study with CR, 45 patients underwent peripheral MRD measurements and revealed a rate of 18 patients with MRD negativity, 6 out of 10 had also no CLL cells detectable in the bone marrow. MRD negativity has been identified as a predictor of ongoing response [6].

These promising results led to the design of the MURANO trial, which compared two time-limited therapies in relapsed/refractory CLL [16]. One arm included rituximab and venetoclax and the other rituximab in combination with bendamustine. Treatment with venetoclax and rituximab showed better response rates and OS rates. Achieving undetectable MRD (defined as < 1 CLL cell/ 10,000 leucocytes; < 10-4) at the end of combination treatment improved PFS and OS compared to patients who had detectable MRD. The MRD-positive group was further divided into two subgroups: MRD low and MRD high positivity. Low MRD positivity was defined with $10^{-4}$ to $<10^{-2}$ CLL cells and high MRD positivity with $>10^{-2}$ revealing significant difference in PFS. Most of the high MRD-positive cases had increasing MRD in peripheral blood before the end of treatment with venetoclax. Apart from MRD they observed del17p, genomic complexity and unmutated IGHV as risk factors for disease progression in patients with undetectable MRD [17]. These data suggest the importance of MRD assessment in time limited therapy strategies before cessation of therapy.

The CLL14 study of the German CLL study group (DCLLSG) led to approval of venetoclax as first-line therapy [7]. This trial investigated a 12-month limited therapy with the Bcl2-inhibitor in combination with the anti-CD20 antibody obinutuzumab and reported a remarkably better PFS than the control group with chlorambucil and obinutuzumab. Three months after treatment completion, more patients in the venetoclax plus obinutuzumab group than in the chloram- bucil plus obinutuzumab group were tested negative for MRD in peripheral blood as well as in bone marrow $(75.5 \%$ vs $35.2 \%$ and $56.9 \%$ vs. $17.1 \%)$. In addition, MRD negativity was consistently more common over all subgroups and more sustainable in patients treated with venetoclax. In CLL14, MRD negativity was assessed by ASO-PCR and confirmed by flow cytometry.

Recent updates with 4 years of follow-up [18] showed a persistence of the PFS advantage and confirmed deeper and more sustainable remissions for patients treated with the Bcl-2 inhibitor compared to chemoimmunotherapy. Nevertheless, a detail which only becomes transparent during longer follow-up is that patients harboring high-risk features may show worse outcomes although treated with venetoclax and obinutuzumab. Results are still superior to obinutuzumab and chlorambucil, but data show that the use of Bcl-2 inhibition alone does not overcome the high-risk features. Whereas patients with mutated IGHV or lacking del17p/TP53, known to be of favorable risk, exhibit the greatest benefit.

Within the patient population of the CLL14 study, the prognostic and predictive impact of genetic markers on achievement of MRD negativity has been analyzed [19]. Three quarters of patients achieved a negative MRD result in peripheral blood when treated with venetoclax and obinutuzumab. Interestingly, in this study, even high-risk genetics like unmutated IGHV, del17p or TP53 mutation had no impact on MRD remission rates. One can conclude that the identified high-risk features affecting the patients' outcome may supersede MRD's predictive power back.

At present, indefinitive therapy with a Bruton tyrosine kinase inhibitor or a limited therapy containing venetoclax are the two most commonly used regimens. Expected upcoming therapies are combination therapies with venetoclax and an inhibitor of Bruton tyrosine kinase, primarily ibrutinib. The laboratory rationale is that ibrutinib and venetoclax act on distinct CLL subpopulations that have different proliferative capacities [20]. While ibrutinib targets mostly the dividing subpopulation of CLL, the resting subpopulation preferentially responds to venetoclax. Therefore, the combination of these two targeted therapies effectively reduced both subpopulations, the resting and the dividing one, in most cases. The TAP CLARITY trial investigated ibrutinib and venetoclax in 54 relapsed/refractory CLL patients with an MRD-driven strategy in stopping the therapy. The overall response rate (ORR) was $94 \%$, with CR/CRi rates of $54 \%$. MRD negativity persisted over time (at month +14 : PB [peripheral blood] 57\% and BM [bone marrow] 39\%) [21].

Recent phase 2 and 3 studies $[22,23]$ as presented at ASCO and EHA this year showed promising results. To avoid tumor lysis syndrome, both studies comprised a lead-in phase with ibrutinib and then a limited 12-cycle combination therapy. One arm of the CAPTIVATE study investigated the fixed duration reg- 
imen without any further therapy, showing promising results, even for patients with del17p or TP53 mutation. ORR were high $(96 \%)$ with more than $50 \%$ CR rates in patients with or without high-risk features. MRD negativity could also be found uniformly distributed in these two groups $(77 \%$ vs $76 \%$ in peripheral blood). Unmeasurable MRD rates were also high in patients with unmutated IGHV status. In the second arm, an MRD-triggered randomization was conducted, administering placebo or ibrutinib for confirmed MRD-negative patients and ibrutinib or the combination therapy (ibrutinib and venetoclax) for not confirmed MRD-negative patients [24]. The results demonstrated that patients with unmeasurable MRD do not benefit from prolonged therapy, showing high response rates even in placebo-treated patients. Otherwise, patients without confirmed MRD negativity showed more additional responses with the combination of ibrutinib and venetoclax in comparison to ibrutinib alone (66\% vs $42 \%)$. Nevertheless, as observation time is relatively short yet, a longer follow-up is needed to confirm these results.

At latest follow-up, 8 patients progressed after end of fixed duration therapy and had been re-treated with single-agent ibrutinib. At least a PR has been achieved in 6 of them [21].

The GLOW study investigated the combination therapy in elderly CLL patients without high-risk features against the standard arm of obinutuzumab and chlorambucil [23]. The CR rates were significantly higher in the investigational arm $(38.7 \%$ vs. $11.4 \%$ ) and more patients reached MRD-negativity (3months after end of treatment $55 \%$ vs $39 \%$ ), respectively. With longer follow-up, patients who received combination therapy with ibrutinib and venetoclax were able to maintain MRD negativity significantly longer (12 months after end of treatment $49 \%$ vs $12 \%$ ) [24]. This means a prolonged time to next treatment for CLL patients with a distinct improvement of quality of life. Interestingly, although patients in this study were significantly older and harbored more comorbidities, the combination regimen was relatively well tolerated.

These studies all demonstrated benefit for the treatment of patients with dell7p over former standard treatment regimens. However, del17p remains a negative prognostic parameter with new agents [19].

These results may push in the direction of an MRDdriven duration of therapy with venetoclax, in combination with an anti-CD20 antibody or a Bruton tyrosine kinase inhibitor. In patients achieving unmeasurable MRD, safe interruption can be recommended with MRD-driven early re-introduction in cases losing response. Nevertheless, this assumes the availability and performance of routine MRD-measurement which will hopefully become standard of care in the near future, at least regarding high-risk CLL patients.

\section{Take home message}

Patients treated with venetoclax can achieve minimal residual disease (MRD) negativity in peripheral blood or bone marrow, whether they receive venetoclaxmonotherapy or in combination with anti-CD20 antibodies. With the upcoming use of combination therapies, MRD negativity will likely become an important marker predicting outcome of chronic lymphocytic leukemia patients.

Funding Open access funding provided by Medical University of Graz.

Conflict of interest K.T. Prochazka and B. Uhl declare that they have no competing interests.

Open Access This article is licensed under a Creative Commons Attribution 4.0 International License, which permits use, sharing, adaptation, distribution and reproduction in any medium or format, as long as you give appropriate credit to the original author(s) and the source, provide a link to the Creative Commons licence, and indicate if changes were made. The images or other third party material in this article are included in the article's Creative Commons licence, unless indicated otherwise in a credit line to the material. If material is not included in the article's Creative Commons licence and your intended use is not permitted by statutory regulation or exceeds the permitted use, you will need to obtain permission directly from the copyright holder. To view a copy of this licence, visit http://creativecommons.org/licenses/by/4.0/.

\section{References}

1. Wendtner CM, Dreger P, Eichhorst B, et al. Onkopedia-Leitlinie Chronische Lymphatische Leukämie (CLL). Stand September 2020. 2020. http:// onkopedia.com/de/onkopedia/guidelines/chronischelymphatische-leukaemie-cll/@@guideline/html/index. html. Accessed: 14.12.2021.

2. Perini GF, Pellegrino Feres CC, Cardoso Teixeira LL, Hamerschlak N. BCL-2 inhibition as treatment for chronic lymphocyticleukemia. CurrTreatOptions Oncol.2021; https:// doi.org/10.1007/s11864-021-00862-z.

3. Del Giudice I, Raponi S, Della Starza I, De Propris MS, Cavalli M, De Novi LA, et al. Minimal residual disease in chronic lymphocytic leukemia: a new goal? Front Oncol. 2019;9:689. https://doi.org/10.3389/fonc.2019.00689.

4. Hanada M, Delia D, Aiello A, et al. bcl-2 gene hypomethylation and high-level expression in B-cell chronic lymphocyticleukemia. Blood. 1993;82(6):1820-8.

5. Roberts AW, Davids MS, Pagel JM, et al. Targeting BCL2 with venetoclax in relapsed chronic lymphocytic leukemia. NEngl J Med. 2016;374(4):311-22.

6. Stilgenbauer S, Eichhorst B, Schetelig J, et al. Venetoclax in relapsed or refractory chronic lymphocytic leukaemia with $17 \mathrm{p}$ deletion: a multicentre, open-label, phase 2 study. Lancet Oncol. 2016;17(6):768-78.

7. Fischer K, Al-Sawaf O, Bahlo J, et al. Venetoclax and obinutuzumab in patients with CLL and coexisting conditions. NEngl J Med. 2019;380(23):2225-36.

8. Rawstron AC, Böttcher S, Letestu R, et al. European Research Initiative in CLL. Improving efficiency and sensitivity: European Research Initiative in CLL (ERIC) update on the international harmonised approach for flow cyto- 
metric residual disease monitoring in CLL. Leukemia. 2013;27:142-9.

9. European Medicines Agency. Guideline on the use of minimal residual disease as an endpoint in chronic lymphocytic leukemia studies. London: European Medicines Agency; 2014. Available online at: http://www.ema. europa.eu/documents/scientific-guideline/guidelineuse-minimal-residue-disease-endpoint-chronic-lymphocytic-leukaemia-studies_en.pdf.

10. Thompson M, Brander D, Nabhan C, et al. Minimal residual disease in chronic lymphocytic leukemia in the era of novel agents a review. JAMAOncol. 2018;4(3):394-400.

11. Wierda WG, Rawstron A, Cymbalista F, et al. Measurable residual disease in chronic lymphocytic leukemia: expert review and consensus recommendations. Leukemia. 2021;35:3059-72.

12. Del Giudice I, Raponi S, Della Starza I, et al. Minimal residual disease in chronic lymphocytic leukemia: A new goal? Front Oncol. 2019;9:689. https://doi.org/10.3389/ fonc. 2019.00689

13. Hallek M, Cheson BD, Catovsky D, et al. iwCLL guidelines for diagnosis, indications for treatment, response assessment, and supportive management of CLL. Blood. 2018;131:2745-60.

14. Rawstron AC, Villamor N, Ritgen M, et al. International standardized approach for flow cytometric residual disease monitoring in chronic lymphocytic leukaemia. Leukemia. 2007;21:956-64.

15. Raponi S, Della Starza I, De Propris MS, et al. Minimal residual disease monitoring in chronic lymphocytic leukaemia patients. A comparative analysis of flow cytometry and ASO IgH RQ-PCR. BrJ Haematol. 2014;166:360-8.

16. Seymour JF, Kipps TJ, Eichhorst B, et al. Venetoclaxrituximab in relapsed or refractory chronic lymphocytic leukemia. NEngl J Med. 2018;378:1107-20.

17. Kater A, Kipps TJ, Eichhorst B, et al. 5-year analysis of Murano study demonstrates enduring uMRD in a subset of R/R CLL patients following fixed-duration VenR. Blood. 2020;136(Supplement 1):19-21.

18. Al-Sawaf O, Zhang C, Rorecht S, et al. Venetoclax-Obinutuzumab for previously untreated chronic lymphocytic leukemia: 4-year follow-up analysis of the randomized
CLL14 study. EHA. 2021;146. Abstract. https://doi.org/10. 1097/HS9.0000000000000566.

19. Tausch E, Schneider C, Robrecht S, et al. Prognostic and predictive impact of genetic markers in patients with CLL treated with obinutuzumab and venetoclax. Blood. 2020;135(26):2402-12.

20. Lu P, Wang S, Franzen C, et al. Ibrutinib and venetoclax target distinct subpopulations of CLL cells: implication for residual disease eradication. Blood Cancer J. 2021;11:39.

21. Hillmen P, Rawstron AC, BrockK, etal. Ibrutinib plus venetoclax in relapsed/refractory chronic lymphocytic leukemia: the CLARITY study. JClin Oncol. 2019;37(30):2722-9.

22. Allan JN, Wierda WG, Sidiqi T, et al. Primary analysis of the fixed-duration cohort from the phase 2 CAPTIVATE study of first-line Ibrutinib + Venetoclax for chronic lymphocytic leukemia/small lymphocytic lymphoma. EHA. 2021;147. Abstract. https://doi.org/10.1097/HS9. 0000000000000566.

23. Kater AP, Owen C, Moreno C, et al. Fixed-duration Ibrutinib and Venetoclax $(\mathrm{I}+\mathrm{V})$ versus Chlorambucil plus Oinutuzumab (CLB+O) for first-line (1L) chronic lymphocytic leukemia (CLL): primary analysis of the phase 3 GLOW study. EHA. 2021;1902. late-breaking abstract. https:// doi. org/10.1097/HS9.0000000000000566.

24. Wierda WG, Tam CS, Allan JN, et al. Ibrutinib (Ibr) plus venetoclax (Ven) for first-line treatment of chronic lymphocytic leukemia (CLL)/small lymphocytic Lymphoma (SLL): 1-year disease-free survival (DFS) results from the MRD cohort of the phase 2 CAPTIVATE study. Blood. 2020;136(Supplement 1):16-7.

Publisher's Note Springer Nature remains neutral with regard to jurisdictional claims in published maps and institutional affiliations.

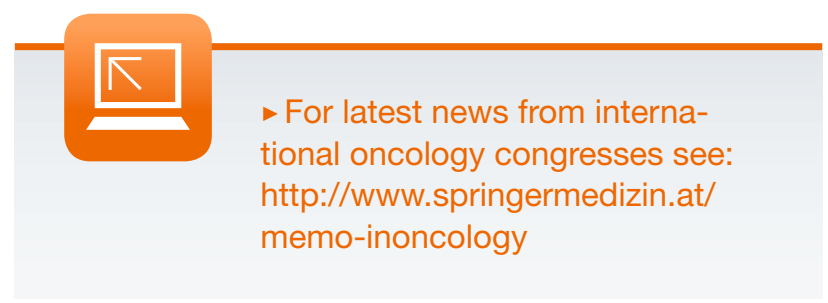

